Revista Digital Año 7. Nº 9 - Año 2016. --pág. 1-104

ISSN 1853-1393

Resistencia. Chaco. Argentina - 2016

\title{
UN DISPOSITIVO DE INTERVENCIÓN-INVESTIGACIÓN CON DIRECTORES DE TESIS DE POSGRADO EN LAS FACULTADES DE CIENCIAS DE LA EDUCACIÓN Y DE CIENCIAS DE LA COMUNICACIÓN DE LA UNIVERSIDAD DE MÁLAGA, DESDE UNA PEDAGOGÍA DOCTORAL
}

\begin{abstract}
AN INTERVENTION-RESEARCH DEVICE WITH POSTGRADUATE THESIS DIRECTORS IN THE FACULTIES OF EDUCATION SCIENCES AND COMMUNICATION SCIENCES OF THE UNIVERSITY OF MALAGA, FROM A DOCTORAL PEDAGOGY
\end{abstract}

Dra. Viviana Mancovsky ${ }^{1}$

Fecha de recepción: 12-08-2016

Fecha de aceptación y versión final: 13-10-2016

Resumen: El presente artículo tiene como propósito relatar una experiencia de trabajo con directores de tesis de posgrado (especialmente del nivel doctoral) a partir de un dispositivo de intervencióninvestigación que se realizó en el Programa de Doctorado en Educación y Comunicación de las Facultades de Ciencias de la Educación y de Ciencias de la Comunicación de la Universidad de Málaga (España). Se llevó a cabo en el marco de una invitación propuesta por la Dra. Nieves Blanco, coordinadora de dicho programa, en noviembre de 2015.

Este dispositivo recupera los aportes teóricos provenientes de una línea de investigación, iniciada en el año 2008, centrada en una Pedagogía Doctoral

La propuesta permitió recuperar las voces de los directores que se animan a cuestionar y "preguntar-se" sobre: en qué consiste su tarea, qué tienen para "dar", cuáles son sus motivaciones para dirigir, qué expectativas tienen del trabajo que realizan y los sentimientos que afloran a lo largo del acompañamiento. Asimismo, mostró la riqueza de pensar de manera entramada la construcción e implementación de dispositivos de intervención y los saberes siempre transitorios, en devenir de una investigación.

Palabras clave: Pedagogía Doctoral. Director de tesis. Análisis de las prácticas profesionales. Saberes de la dirección. Dispositivo de intervención-investigación.

\begin{abstract}
This article aims to relate an experience of working with directors of postgraduate thesis (especially from the doctoral level) from an intervention-research device that was carried out in the Doctoral Program in Education and Communication of the Faculties of Sciences of The Education and Communication Sciences of the University of Málaga (Spain). It was accomplished in the framework of an invitation proposed by Dr. Nieves Blanco, program coordinator, in November 2015.

This device recovers the theoretical contributions of a research line, initiated in 2008, centered on a Doctorate Pedagogy. The idea allowed to recover the voices of the directors who are encouraged to question and "ask themselves" about: what their task consists of, what they have to "give", what their motivations to lead are, what expectations on the work they perform they have, and the feelings that arise through the accompaniment.

Likewise, it showed the richness thinking in a structured way the construction and implementation of intervention devices and the ever transient knowledge.

Likewise, the richness of thought showed in a structured way the construction and implementation of intervention devices and the ever transient knowledge, in the making of an investigation.
\end{abstract}

Key words: Doctoral. Pedagogy - Thesis director - Analysis of professional practices - .Knowing the address. Intervention-research device -

\footnotetext{
1 Pertenencia Institucional: Universidad Nacional de Tres de Febrero y Universidad Nacional de San Martín. Domicilio: Felipe Vallese 1301 (CP: 1405) CABA. Teléfono: 15337902 04. Correo electrónico: vivmanco@yaho.com.ar.
} 


\section{Presentación}

Este artículo tiene como propósito relatar una experiencia de trabajo con directores de tesis de posgrado (especialmente del nivel doctoral) a partir de un dispositivo de intervención-investigación que se realizó en el Programa de Doctorado en Educación y Comunicación de las Facultades de Ciencias de la Educación y de Ciencias de la Comunicación de la Universidad de Málaga (España). Se llevó a cabo en el marco de una invitación propuesta por la Dra. Nieves Blanco, coordinadora de dicho programa, en noviembre de 2015.

El dispositivo recupera los aportes teóricos provenientes de una línea de investigación, iniciada desde el año 2008, centrada en una Pedagogía Doctoral. Tiene como finalidad "generar las condiciones" para favorecer el diálogo entre directores de posgrado acerca de la compleja y delicada tarea que supone acompañar el proceso formativo, a largo plazo, de los doctorandos. En este relato, me interesa poner en relieve la voz de los protagonistas como un modo de transmitir sus apreciaciones y puntos de vista, dar visibilidad a su tarea y reconocer la necesidad de crear formalmente espacios institucionales de encuentro e intercambio.

De manera introductoria, considero necesario presentar la noción de "dispositivo de intervención" desde el campo de la formación de adultos que pone hincapié en los aprendizajes relativos al saber y al saber-hacer profesional. Este término va más allá de concebir estrategias y técnicas, a la manera de un diseño de acciones relacionadas entre sí y planificadas de antemano. Supone una organización de prácticas, medios y recursos que apuntan a "crear las condiciones" para que los sujetos participantes del mismo, alcancen determinados propósitos en situaciones concretas. Generalmente, desde este campo, los objetivos responden a fines formativos.

Por su parte, la palabra "intervención", en el marco de un trabajo con sujetos, prácticas e instituciones, encierra algunos sentidos fundamentales: intervenir como un "venir y estar entre", "como posibilidad de transformar y favorecer cambios" y como "consideración de lo que acontece". Específicamente, desde un enfoque psicosocial y en el marco de una institución, "....intervenir supone permitir a las personas moverse, pensar de otra manera, hacerse nuevas preguntas y planear otras relaciones..." (Correa, 2004: 13)

La posibilidad de crear e implementar dispositivos de intervención con una población de estudio a partir de los avances que propone la Pedagogía Doctoral traduce una manera de concebir a la producción teórica en permanente construcción. No se trata de una simple aplicación de resultados investigativos. Por el contrario, el hecho de vincular la intervención y la investigación de manera entramada supone que "...cada intervención específica presenta el desafío de interrogar certezas teóricas e inventar nuevos diseños de intervención”. (Fernández y cols. 2005: 13)

De este modo, el trabajo se organiza en un primer apartado en el cual me propongo describir el recorrido de la Pedagogía Doctoral desde las primeras preguntas, las delimitaciones del objeto de estudio, los intercambios con diferentes equipos de investigación y las producciones teóricas elaboradas, fruto de los resultados alcanzados hasta el presente. Luego, relataré el dispositivo de intervencióninvestigación recuperando la voz de los directores participantes que asisten a a propuesta. Por último, formularé algunas reflexiones finales en torno a nuevas preguntas e hipótesis para continuar este devenir teórico "en permanente construcción".

\section{Breve recorrido de la Pedagogía Doctoral desde los inicios.}

Desde hace casi unos diez años, comencé a interesarme por la diversidad de problemáticas que se despliegan en torno a la formación doctoral de los sujetos que 
deciden seguir este nivel de posgrado académico. En ese marco, avancé paulatinamente hacia la construcción de un escenario de reflexión posible tendiente a dar visibilidad el entramado de dimensiones de análisis que atraviesa dicho nivel formativo. Estas dimensiones comprenden diferentes aspectos: personales y subjetivos, vinculares, institucionales, curriculares (según un programa doctoral determinado), de política educativa del nivel de posgrado y más ampliamente, relativos a los vastos campos disciplinares del conocimiento. Dicho de otro modo, intenté poner en evidencia que el doctorado puede analizarse desde el punto de vista de: los sujetos implicados (los responsables de un programa, el doctorando y las interacciones entre pares, el director de tesis, los profesores que conforman comisiones de admisión, asesoramiento y/o dictan seminarios, los miembros de un jurado), las instituciones y los programas que ofrecen, la política educativa que lo enmarca y los marcos disciplinares que condicionan estilos formativos diversos según las características propias del objeto de conocimiento.'.

Así, formulé algunas primeras preguntas sobre la formación doctoral intentando analizar las condiciones institucionales y los desafíos intelectuales y personales que se ponen en juego de parte del sujeto que transita un determinado programa doctoralii. Específicamente, la noción de relación con el saber me ayudó a poner en evidencia la dimensión subjetiva que traduce una experiencia formativa singular a partir de la cual, el sujeto "se autoriza" a crear saberes nuevos argumentando académicamente el proceso de investigación que fundamenta dicha creación. En este sentido, la tesis doctoral no solo expone la investigación realizada sino que también da "testimonio" de los cambios identitarios que atraviesa el doctorando en vistas a convertirse en "autor" de los saberes que produce (Mancovsky, 2009). El análisis de las entrevistas tomadas a estudiantes de doctorado y a doctorados "recientes" dieron cuenta de sus motivaciones a partir de las cuales se aventuran a dicho proyecto, sus temores, las dificultades encontradas y las vicisitudes que atraviesan a lo largo de su experiencia formativa.

Luego, puse hincapié en el estudio de la relación formativa entre director de tesis y tesista desde el "mutuo acuerdo" que traduce una supuesta elección entre ambos. Comencé por preguntarme acerca de la naturaleza de ese vínculo intersubjetivo que se despliega en el tiempo y entrama motivaciones, intereses, propósitos, saberes, emociones y sentimientos tanto de parte del sujeto en formación como del director.

Poco a poco, a partir de los resultados de las investigaciones realizadas, basados en entrevistas a directores de programas institucionales, directores de tesis y a doctorandos de distintos contextos institucionales y de universidades de diversos países, fui pensando en la posibilidad de nombrar a ese escenario de reflexión como un incipiente campo dentro de la Pedagogía de la Formación, reconociendo la especificidad del nivel. En este sentido, la expresión Pedagogía Doctoral empezó a cobrar identidad en mis trabajos de investigación y en mis intercambios entre colegas. Inicialmente, dicha expresión fue enunciada con todos los recaudos teóricos y hasta con cierto temor personal, sabiendo de las dificultades y las resistencias por otorgar un lugar a la pedagogía en la universidad y sobre todo, animarme a usarla para el nivel de mayor formación académica. Cuando la pedagogía suele ser asociada a lo escolar y a los aprendizajes de la infancia, ¿cómo atreverse a pensarla en el mundo de la formación de adultos y encima, para el ámbito de "la academia"? Al día de hoy, esta expresión sigue siendo rechazada entre algunos grupos de investigadores de pertenencia institucional diversa ${ }^{\text {iii }}$.

Por otra parte, el intercambio con diferentes equipos de investigación interesados en estos temas afianzó mi interés y a su vez, fue legitimando el hecho de autorizarme a usar la expresión con mayor convicción. Más precisamente, el trabajo entablado con la Dra. M. G. Moreno Bayardo y su equipo de investigadores, en la 
Universidad de Guadalajara, sirvió de antecedente a una obra en co-autoría, publicada en el 2015, titulada: La formación para la investigación en el posgrado. Dicho trabajo concluye reconociendo el lugar clave de los diversos y variados aprendizajes que realiza un sujeto que se forma como investigador. Desde esta afirmación, sostengo la pertinencia de un abordaje pedagógico como un espacio de reflexión que indaga cuestiones relativas a dichos procesos de aprendizaje, los acompañamientos formativos necesarios y las condiciones institucionales para que se desarrollen. Más precisamente, identifico como posibles problemáticas "pedagógicas" del nivel doctoral a:

- los saberes que el director pone en juego cuando lleva a cabo su práctica de acompañar un tesista y corregir sus producciones dando cuenta de los aprendizajes alcanzados,

-el posible seguimiento institucional de la relación director de tesis y doctorando, tesis,

-el estudio de los casos de los estudiantes que abandonan y no terminan sus

-las instancias curriculares específicas de formar para la investigación,

-el análisis de las estrategias previstas en un programa institucional dedicadas al intercambio de los avances de las investigaciones (coloquios, ateneos, etc.),

-el estudio sobre el modo en que se llevan a cabo las defensas de tesis (desde la selección y conformación del jurado hasta la instancia de concreción y los posteriores comentarios del sujeto recientemente doctorado), entre otras.

En este contexto, me interesó profundizar el estudio de la práctica del director de tesis dado que suele ser una tarea que asume características particulares, a saber:

-se da como algo esperado dentro del conjunto de responsabilidades y tareas docentes en la universidad, reconociéndose muchas veces como una exigencia más, "algo que te toca"...

-se ejerce casi sin incumbencia de la institución formadora,

-se "aprende" por el solo hecho de haber pasado por la experiencia de haber sido dirigido, de manera casi "natural", repitiendo el modelo de dirección vivido o intentando hacer todo lo contrario,

Se suman además, otros comentarios informales acerca de la tarea de dirigir, que traducen la necesidad de describirla recuperando el punto de vista de los propios directores: el tiempo que insume, la dedicación, las satisfacciones que produce y las dificultades que van apareciendo en cada relación formativa singular con cada doctorando, los niveles de exigencia, el producir una tesis "de calidad", las incertidumbres acerca de los distintos momentos por los que atraviesa el tesista. Por todos estos motivos, junto con mis propias preguntas personales acerca de mi tarea como directora de tesis y el análisis de mi implicación en dicha actividad, decidí adentrarme en el estudio de la relación formativa entre director de tesis y doctorando desde la perspectiva de los directores. A partir de un trabajo empírico basado en entrevistas en profundidad, pude realizar un intento de clasificación acerca de los 
diversos saberes que un director despliega en el acompañamiento de un tesista (Mancovsky, 2013). Entre ellos, pude identificar los siguientes:

-Saberes relativos a un campo científico determinado, a partir de los cuales, el objeto de investigación acordado entre tesista y director, se corresponde en mayor o en menor medida, con la especialidad y el interés de estudio de este último. Esos saberes se suponen referentes clave de la tarea de dirigir y muchas veces, estructuran los primeros acuerdos que definen la relación formativa.

-Saberes vinculados con las exigencias propias de la escritura académica que debe transmitir un director a un doctorando acerca de la especificidad de un género discursivo altamente codificado y a partir de los cuales, lo acompaña en la construcción de un nuevo "posicionamiento enunciativo" vinculado al convertirse paulatinamente en "autor-productor de conocimiento". (Arnoux, E. y otros, 2005).

-Saberes referidos al aprendizaje del oficio de investigador que comprenden el conjunto de habilidades cognitivas a desarrollar durante la formación para la investigación del tesista, como lo describe Moreno Bayardo (2015). Dichos saberes dan cuenta de la complejidad de la tarea del director fomentando y a su vez, exigiendo el aprendizaje de estas habilidades por parte del sujeto en formación.

-Saberes relativos al acompañamiento de la socialización académica del doctorando. Suponen el manejo y la transmisión de las "reglas de uso" que encierra el aprender a participar en una comunidad científica.

-Saberes "relacionales" que ponen en relieve las cuestiones subjetivas y vinculares del acompañamiento y sostienen el proceso de cambio identitario del doctorando. El director despliega este tipo de saberes de manera más o menos intuitiva, lo sepa o no, lo problematice o no, más allá de los resultados logrados.

De esta manera, a partir de las investigaciones realizadas y de los diversos encuentros con colegas investigadores de diferentes universidades en los cuales compartimos preocupaciones comunes acerca de la formación doctoral, ya sea como directora de tesis o como profesora de seminarios de doctorado, comencé a crear y a llevar a cabo distintas prácticas de intervención - investigación para problematizar institucionalmente algunos de los temas que fundamentan una Pedagogía Doctoral (Mancovsky, 2014a, 2014b). En el siguiente apartado, me propongo relatar precisamente la implementación de uno de los dispositivos creados, destinado a directores de tesis de posgrado.

\section{Un dispositivo de intervención-investigación en el marco de un seminario-taller destinado a directores de tesis}

Antes de presentar el dispositivo, considero necesario exponer brevemente algunos aspectos teóricos que encuadran y fundamentan el sentido de la propuesta desde la perspectiva del "análisis de las prácticas profesionales", enmarcada en el campo de la Pedagogía de la Formación.

A modo de introducción y recuperando los usos comunes de la expresión, el análisis de las prácticas comprende la posibilidad de reflexionar sobre "lo que se hace", la propia tarea realizada, los aciertos, las dificultades, los imprevistos que aparecen en el día a día, los saberes que guían las decisiones a tomar y "los trucos" del oficio que se aprenden con el desempeño de una tarea, la "toma de distancia" para poder ver lo sucedido y el grado de satisfacción o insatisfacción de lo realizado, el 
"deber ser" y los logros alcanzados. De manera más o menos espontánea, intuitiva y/o consciente, es común encontrar esta instancia de reflexión en el desempeño de una tarea o de un trabajo.

Ahora bien, en el ámbito profesional, bajo la denominación "análisis de las prácticas" se agrupan diversas propuestas que responden a propósitos diferentes. Algunos de ellos pueden estar vinculados con: identificar y explicitar las competencias que sostienen una práctica, querer evaluarla, conformar una herramienta de intervención institucional, seleccionar modelos de "buenas prácticas" para darlos a conocer y difundirlos, capacitar y formar a profesionales, entre otros.

A su vez, el análisis de las prácticas se fundamenta en marcos teóricos diversos que condicionan dichos fines y orientan una implementación específica. Algunos de esos abordajes teóricos son propuestos desde: la didáctica, el psicoanálisis, la psicosociología, la psicología del trabajo y/o la psicología cognitiva. Asimismo, los dispositivos y las propuestas se diseñan y se realizan en muy variados ámbitos como ser los de: la salud, la educación, el trabajo social o la empresa.

Intentando precisar una primera definición, J. Chaim y C. Humbert (2014), ambos psicosociólogos, sostienen que el "análisis de las prácticas" es un método de conocimiento y de formación; no de cura terapéutica. Es un dispositivo de intervención que permite construir saber y saber-hacer sobre la propia manera de ejercer una profesión. Remitirse al término "análisis" no involucra, directamente al abordaje psicoanalítico. El sentido genuino del término se remite a un ejercicio de distinción y de identificación. Básicamente, reafirman los autores, el análisis de las prácticas es un trabajo de reconocimiento, de búsqueda de sentido y de comprensión. Más que una reflexión sobre "lo que se debe hacer" o "lo que se irá a hacer", el análisis de las prácticas se basa sobre "lo hecho" y en la capacidad de reconstruir y hacer de ese análisis, una experiencia con sentido formativo.

Por su parte, una definición que complementa la anterior, la aportan dos autores desde la Pedagogía de la Formación. C. Blanchard-Laville y D. Fablet (1996) definen al análisis de prácticas como: “...una actividad de formación, organizada en un marco instituido de formación profesional, inicial o continua, concerniente a profesionales que ejercen oficios (formadores, docentes, trabajadores sociales, psicólogos, terapeutas, médicos, responsables de recursos humanos...) o funciones incluyendo dimensiones relacionales importantes, dentro de campos diversificados (de la educación, de lo social, de la empresa...); estableciendo dispositivos en los cuales los sujetos son invitados a implicarse en el análisis, es decir a trabajar en la co-construcción del sentido de sus prácticas, y/o a mejorar las técnicas profesionales; conduciendo ello a una elaboración en situación interindividual, lo más a menudo grupal que se inscribe dentro de cierta duración y requiere la presencia de un animador, él mismo, en general, profesional del área de las prácticas analizadas, garante del dispositivo vinculado con referencias teóricas afirmadas"... "(C. Blanchard-Laville y D. Fablet, 1996).

Dentro de este campo de la Pedagogía de la Formación, me interesa subrayar la importancia de construir dispositivos de intervención-investigación que habiliten espacios de encuentro, intercambio y discusión entre colegas que cumplen una misma tarea. La propuesta recupera teóricamente algunos de los rasgos que se derivan del "análisis de las prácticas profesionales". El relato del dispositivo no consiste en la exposición de una simple actividad pedagógica planificada y realizada en un marco institucional determinado sino que, por el contrario, intenta poner en evidencia la necesidad de fomentar el encuentro entre profesores, profesionales que hacen lo misma tarea, tienen las mismas responsabilidades, ponen en juego un conjunto de estrategias, una dedicación y un bagaje de conocimientos en torno a una práctica común: la dirección de tesis de doctorado. Si bien es un dispositivo que "interviene" institucionalmente convocando la participación de un grupo específico de actores 
institucionales, los directores de tesis, también aspira a hacer avanzar los resultados, siempre transitorios, de la investigación que realizo en torno a una Pedagogía Doctoral desde hace casi una década.

El dispositivo se llevó a cabo en las Facultades de Ciencias de la Educación y de Ciencias de la Comunicación de la Universidad de Málaga en acuerdo con la coordinación del Programa de Doctorado en Educación y Comunicación. Se lo concibió como un Seminario-taller destinado a directores y directoras de tesis doctorales. Se invitó también, a aquellos y aquellas profesores/as que dirigían trabajos finales de master de las distintas unidades académicas de la Universidad. Llevó por título: "La complejidad de la relación formativa en la dirección de tesis: tensiones, saberes, retos". Tuvo una duración estimada de tres horas. Su propósito general fue explicitado del siguiente modo: crear un espacio de intercambio entre profesores que dirigen tesis de posgrado a fin de identificar algunos aspectos relevantes de la tarea, en términos de "apoyo-sostén" y/u "obstáculo-dificultad".

Los ejes temáticos que enmarcaron las consignas de trabajo del Seminario-taller fueron derivados de los resultados de mis investigaciones y se formularon como:

-El aprendizaje del "oficio de investigar": la transformación subjetiva del doctorando que atraviesa este proceso formativo.

-La relación formativa que se construye entre director y doctorando: la inevitable tensión entre la autorización y la autoría.

-Los saberes que se ponen en juego:

- disciplinares,

- relativos a la escritura académica,

- vinculados a la metodología de investigación,

- en relación con la socialización académica y

- relacionales, relativos al "sí mismo" del tesista y del director.

A partir de este programa, se realizó la difusión del seminario-taller en las distintas unidades académicas de la Universidad fijando lugar, día y hora de encuentro.

Asistieron diecisiete directores pertenecientes a las Facultades de: Ciencias de la Educación, Ciencias de la Salud, Ciencias Económicas y Empresariales y Ciencias de la Comunicación. Es necesario mencionar que es la primera vez que se organiza una actividad que convoque a los directores de tesis de posgrado al tratamiento y a la discusión de estas temáticas específicas sobre el desempeño de su rol.

Ahora bien, para relatar la puesta en marcha del Seminario-taller con los directores, necesito presentar una de las primeras actividades realizadas en esta semana de trabajo. Los dos primeros días estuvieron dedicados al intercambio con los doctorandos de diferentes programas doctorales de la Universidad. El fundamento de incluir lo trabajado con los doctorandos es que, parte de lo producido en esos encuentros, fue insumo para la discusión en el seminario-taller con los directores.

Específicamente, se invitó a participar a los estudiantes del Doctorado en Educación y Comunicación Social de la Facultad de Ciencias de la Educación. Este encuentro llevó por título "Aprender el oficio de investigador/a. experiencias y saberes en el camino hacia la autonomía intelectual". Debido a la gran cantidad de 
participantes que se inscribieron, se acordó desdoblar la actividad en dos días ya que la intención fue trabajar en pequeños grupos y favorecer el intercambio entre pares acerca de cuestiones específicas de su experiencia formativa. Por tal motivo, la misma propuesta se replicó en dos días consecutivos para dos grupos diferentes de doctorandos. En cada uno de los encuentros, asistieron alrededor de 30 participantes. Es de destacar que, en la segunda jornada, se incluyeron doctorandos de otros programas.

Las consignas de trabajo para cada grupo versaron sobre diferentes ejes temáticos que, a su vez, encerraban una pequeña lista de preguntas disparadoras, a saber:

-1er. grupo: "Expectativas": ¿Qué esperás del doctorado? ¿Qué crees que puedes aportar?

$-2^{\circ}$ grupo: "La relación formativa con el director": ¿Qué esperás de tu director o tu directora? ¿Qué expectativas tenés de esa relación?

$-3^{\circ}$ grupo: "El sentido de una tesis doctoral": ¿Qué es una tesis para vos? ¿Por qué hacés una tesis?

-4 grupo: "Cuestiones personales": ¿Qué te entusiasma o te anima en este proceso de formación? ¿Qué temores tienes y qué te preocupa?

$-5^{\circ}$ grupo: "Intercambios con otros sobre el propio proceso formativo": ¿Con quién hablas acerca de lo cotidiano de tu estudio? (Tus temores, tus dudas, tus hallazgos, tus aciertos, etc.)

Cada encuentro tuvo una duración de tres horas. En el inicio, se llevó a cabo una primera actividad colectiva estimulando la participación de todos los doctorandos presentes, a partir del análisis de unas imágenes que representaban esculturas de la figura humana en diferentes posiciones y sobre todo, de la cabeza ${ }^{i v}$. Este ejercicio de caldeamiento grupal alentó la toma de la palabra y el pensamiento libre sobre "la propia experiencia de formación doctoral", desde el uso de un recurso artístico.

Luego, se dio la consigna de trabajo en pequeños grupos. En un tercer momento, se realizó la puesta en común donde se socializaron las respuestas.

Las opiniones y las apreciaciones compartidas en los pequeños grupos fueron recogidos por escrito, respetando el anonimato y formaron parte del material inicial que abrió el debate y la participación en el seminario-taller con los directores.

Seleccioné algunas de esas valiosas intervenciones de los doctorandos para introducir la problemática sobre la "relación formativa entre director y tesista" desde las expectativas de este último, a saber:

-"El director debe tener un compromiso con la formación del doctorando, no solo con la adquisición o el logro de los resultados en la investigación".

“¿Debe existir complicidad y apoyo de parte de tu director?"

-"El director es un punto de contacto con la comunidad investigadora, una guía de aprendizaje, una ayuda para la superación de los bloqueos, un activador del pensamiento".

-"Es una relación muy cercana, muy amiga”. 
-"La comunicación debe ser buena. No es tanto verse todos los días sino tener una comunicación fluida. Tener acceso a la directora..."

-"El director te indica por donde debes ir pero la última palabra la tengo siempre yo. Al final, decido yo".

-"El director te ayuda a acotar, a centrarte en un tema".

-"El director debe creer en tí. Que vea en tí ese futuro investigador que queréis llegar a ser, a investigar como una forma de vida".

-"Yo también tengo que creer en el director".

-"El director debe querer compartir experiencias contigo, dedicarte tiempo". indiferente".

-"Es importante que el director se entusiasme con tu tema, que no le sea

-“A mí me interesa tener solo una relación académica, no personal, con mi director".

-"Espero compromiso de parte de mi director. A veces, están sobrecargados de tarea y lo que para nosotros es una prioridad como un encuentro o responder un correo, para ellos, no".

-"Espero reconocimiento y confrontación de ideas diferentes".

-"Espero claridad de objetivos y llegar a un consenso".

Resulta interesante observar cómo las opiniones de los doctorandos sobre la tarea del director dejan entrever los supuestos saberes que éste pone en juego en torno a: lo disciplinar relativo al objeto de investigación, la socialización académica y la participación en una comunidad científica, el aprender el oficio de la investigación y el cuidado del vínculo (por ejemplo: la confianza, el estilo comunicacional, la frecuencia, la conquista de autonomía, entre otras preocupaciones abordadas por los doctorandos).

En el cierre de la puesta en común con los doctorandos, puse hincapié en pensar estas cuestiones como "puntos extremos de posibles tensiones" que caracterizan a toda relación formativa: ¿confianza-desconfianza, dependenciaautonomía, cercanía-distancia, relación académica-relación amiga, reconocimientoindiferencia, dedicación-ausencia? Llegamos a concebir conjuntamente a la relación formativa como un proceso que se construye en el largo plazo con el director y que atraviesa distintas etapas en relación con "los aprendizajes" que supone la puesta en marcha de una investigación y la escritura respectiva de la tesis. Pudimos tematizar que, en el transcurrir de ese proceso, el doctorando experimenta cambios intelectuales en su manera de pensar, argumentar, estudiar, abordar el trabajo empírico, escribir, sistematizar lecturas e ideas propias, etc. que conllevan a su vez, a cambios identitarios significativos.

Con todo este material producido por los doctorandos en los pequeños grupos, recogido y ordenado, inicié el intercambio con los directores de tesis en el Seminariotaller. La intención fue retomar las opiniones dadas por estudiantes para ser analizadas desde el punto de vista de los directores, consideradas como "disparadoras de la discusión". 
Comencé el encuentro contándoles a los participantes las actividades realizadas con los doctorandos. Especialmente, me detuve en compartir con ellos las opiniones dadas en los pequeños grupos sobre los distintos ejes temáticos. Los directores se mostraron sumamente sorprendidos al escuchar estas apreciaciones y se dispusieron al debate, planteando sus acuerdos y sus desacuerdos a partir de lo que más les llamó la atención. Así, fueron expresando sus propias reflexiones que intentaré transmitir en esta breve recopilación, a partir de mis registros escritos, elaborados a medida que transcurría el intercambio colectivo:

-“Orientar es un proceso muy, muy importante y requiere mucha dedicación. Para mí, es un trabajo muy intenso. Construir un objeto de estudio, trabajarlo, pulirlo... lleva mucho acompañamiento cara a cara... por lo menos en los comienzos, lleva mucho tiempo".

-"Yo parto por identificar un interés de parte del estudiante, un compromiso... Sobre eso, armamos un contrato inicial y yo planteo mi exigencia..."

-"La responsabilidad final debe recaer en el doctorando..." debe..."

-"Es difícil dar autonomía a alguien que no está haciendo las cosas como

-"Yo creo que hay que hacerse una pregunta central, de fondo: ¿por qué queremos dirigir tesis?"

- ¿Necesitamos mano de obra o tenemos, reconocemos un alumno bueno y queremos sacarlo mejor?"

¿¿¿Qué tengo yo para ofrecer?”

-"No es fácil acompañar un proceso de superación personal y profesional que tiene que venir desde la decisión de cada uno"...

-“Dirigir, a veces, es una carga... Puede ser visto como algo negativo..."

-"Algunos aceptan doctorandos y no se hacen cargo. Se sienten superados en el número de los tesistas que dirigen..."

-"Hay tesistas que solo quieren de uno, el acuerdo inicial y la firma final para presentar la tesis. No todos están dispuestos a construir una relación y dejarse orientar..."

-“Con cada tesista es una relación nueva. Te puede ir bien ... como no tanto...”

-"Es agotadora la instancia de corrección de borradores y más, si ya hay una fecha establecida de presentación de tesis..."

-"Da mucha satisfacción ver el proceso de un tesista que aprende a argumentar bien sus decisiones"...

Así como en la puesta en común con los doctorandos aparecieron las expectativas puestas sobre "los supuestos saberes" de un director, en estas intervenciones recopiladas de los directores surge como relevante la necesidad de 
"pensarse haciendo", hablar sobre "lo que uno hace cuando dirige". Estas opiniones contienen, de manera explícita o implícita, las sensaciones que ellos mismos perciben en torno a ese hacer. Algunas palabras que resuenan en este registro de lo sensible son: satisfacción, agotamiento, carga, dificultad, intensidad, incertidumbre, entre otros. También, de manera menos explícita, puede sentirse cierto malestar o enojo.

Me resulta sumamente significativo que en el intercambio entre colegas haya aparecido la pregunta acerca del sentido sobre la tarea de dirigir: el por qué. También, en estas opiniones, aparecen evidenciadas "las tensiones" de la relación formativa entre la responsabilidad de uno y/o de otro, la dedicación y el compromiso, como en los intercambios entre doctorandos.

Hay un tema clave de la relación formativa que aparece sin ser abordado en profundidad y que pongo en evidencia a partir de haber implementado este dispositivo con directores. El mismo alude a la referencia permanente al "tiempo" como factor clave para encuadrar la tarea de director: la dedicación, la diversas de tareas que realiza como la corrección de borradores, la frecuencia de los encuentros, la presión de los plazos de entrega, etc.

Este dispositivo de intervención permitió recuperar las voces de los directores que se animan a revisarse y pensar sobre: en qué consiste su tarea, qué tienen para "dar", cuáles son sus motivaciones para dirigir, qué expectativas tienen del trabajo que realizan y los sentimientos que afloran a lo largo del acompañamiento. Si bien algunas opiniones estuvieron más centradas en "el afuera", en poner la exigencia en lo que "el doctorando puede o debe hacer", otras intervenciones se dispusieron a "trabajar sobre sí mismos", a verse y a interrogarse.

En síntesis, las opiniones recogidas me resultan sumamente relevantes para argumentar a favor de crear dispositivos de intervención que alienten la posibilidad de revisar y comprender los sentidos que construyen los directores acerca de la tarea que realizan. Dichas opiniones han dado lugar a vislumbrar aspectos facilitadores $u$ obstaculizadores de la tarea, como se formuló en el propósito general de la propuesta. Específicamente, en relación a las dificultades que aparecen, sostengo que el hecho de poder enunciarlas identificando las limitaciones y los condicionantes frente a una situación dada y pensada junto con otros, ayuda a la búsqueda de posibles alternativas y soluciones. Los dispositivos de intervención aspiran precisamente a eso.

Por último, es de destacar que los comentarios finales de los directores de tesis acerca del encuentro compartido pusieron de manifiesto la necesidad de fomentar y facilitar regularmente espacios institucionales para debatir y analizar, junto con otros, los aciertos y las dificultades propias de quienes ejercen la práctica de dirigir.

\section{A modo de reflexión final}

En principio, la evaluación del dispositivo implementado tiene que ver con la posibilidad de "crear las condiciones" para que los directores piensen y se piensen juntos. Más precisamente, las consignas previstas han tratado de intervenir sobre una práctica "naturalizada" y poco "compartida" entre los directores de tesis. Práctica que suele describir un trabajo prolongado en el tiempo, en soledad, más allá del encuadre de la relación formativa con el tesista. Práctica que muchas veces suele ser vivida desde la satisfacción y el agrado pero también, desde el agobio, el malestar o el "no saber directamente qué hacer".

A partir del análisis del dispositivo de intervención realizado, observo un vínculo directo con la investigación al pensarlo como una propuesta que retroalimenta al objeto de estudio centrado en: el director de tesis y los saberes que pone en juego en la relación formativa con el doctorando. Me resulta valioso plantear esta complementariedad: cómo la intervención ofrece un material empírico para ser analizado desde la investigación y a la inversa. Esta evidencia me permite 
fundamentar la necesidad de crear y diseñar otros dispositivos de intervención que fomenten el intercambio sobre "el análisis de la práctica de dirigir" con directores de tesis. Sería deseable instalar dispositivos permanentes que aseguren las condiciones institucionales para el encuentro en un tiempo-espacio regular, a partir de un estilo de coordinación singular. Un programa doctoral podría ofrecer esta posibilidad, instalando un dispositivo de análisis de las prácticas de dirección de manera estable. Seguramente, el trabajo colectivo de los directores, en esta línea, redundará en relaciones formativas más productivas y "flexibles" con los doctorandos. Esto a su vez, podría mejorar el índice de terminalidad de las tesis.

De esta manera, tanto la investigación como la intervención podrían entramarse con un espacio institucional de formación continua de los directores de tesis. Una formación que permita intercambiar con otros colegas las preguntas, las dudas, los aciertos, los imprevistos que aparecen en la práctica de dirigir. Una formación que fomente el construir colectivamente saber y saber-hacer sobre dicha práctica. Una formación que acompañe un cuestionamiento sobre sí mismo, sobre sus motivaciones y la tensión autoridad-autonomía que siempre se manifiesta en toda relación formativa.

Por último, la posibilidad de diseñar y llevar a cabo institucionalmente dispositivos de intervención-investigación-formación requiere de un estilo de coordinación que encuadre un trabajo cuidadoso. Dicha coordinación se aleja de una actitud crítica y prescriptiva que va en desmedro de la confianza necesaria para reducir las resistencias iniciales de los participantes que suele provocar una propuesta de esta naturaleza. Este estilo, también, supone una disposición a incluir "lo que acontece" en el intercambio entre colegas. Desarrolla una atención particular a los vínculos entre los participantes respetando la singularidad y la capacidad de transformación y de aprendizaje de cada uno. Además, despliega una escucha atenta y cualitativamente paciente. Asimismo, la coordinación de un dispositivo de análisis de prácticas incluye una consideración hacia los aspectos afectivos, conscientes e inconscientes, que influyen en interacciones de los actores involucrados en la propuesta. Por último, exige un trabajo imprescindible con la propia implicación de parte de la coordinación del dispositivo. Este análisis no responde a un fin terapéutico. Más bien, apunta a una reflexión subjetiva y ético-política desde el conocimiento de sí mismo que desnaturaliza supuestos, creencias y prejuicios. Todos estos rasgos de la coordinación pueden situarse dentro del enfoque clínico en las ciencias sociales (Mancovsky, V. 2011).

Considero, finalmente que la posibilidad de llevar adelante esta propuesta centrada en el análisis de las prácticas de los directores de tesis desde un enfoque clínico habilita un modo de disponerse al trabajo con el otro que puede redundar en un enriquecimiento de la relación formativa que el director entabla con cada doctorando. Probablemente, la posibilidad de vivenciar las cualidades de la "modalidad clínica" de la coordinación, a partir de la participación del director en el dispositivo, podría redundar en su propio estilo de dirección que despliega en la relación formativa con cada doctorando. Esta afirmación se convierte en una valiosa y sugerente hipótesis a seguir indagando en nuevas investigaciones.

\section{Bibliografía}

Blanchard-Laville, C. et Fablet, D. (1996) L'analyse des pratiques professionnelles. Paris. L`Harmattan.

Beillerot, J. (1996) La formación de formadores. Buenos Aires. Ediciones Novedades Educativas y Facultad de Filosofía y Letras.

Correa, A. (2004) "Apertura del Seminario: La intervención psicosocial”, En: Cuadernos del Campo Psicosocial, №. 2. Universidad Nacional de Córdoba. 
Chaim, J. et Humbert, C. (2014) Dispositifs d'analyse des pratiques et d'intervention. Paris. L'Harmattan.

De la Fare, M. y Lenz, S. (2012) El posgrado en el campo universitario. Expansión de carreras y productividad de tesis en la Argentina. Universidad Nacional de General Sarmiento. IEC-CONADU.

Fernández, A. "Los asedios a la imaginación" Nota publicada en el Diario Página 12. 24 de marzo, del 2000. Buenos Aires. Argentina.

Mancovsky, V. (2009). "¿Qué se espera de una tesis de doctorado? Breve introducción sobre algunas cuestiones en torno a la formación doctoral". En: Revista Argentina de Educación Superior. № 1. Noviembre, 2009. Red Argentina de Posgrados en Educación Superior. Edición digital: www.untref.edu.ar/raes

Mancovsky, V. (2013). "La dirección de tesis de doctorado: Tras las huellas de los saberes puestos en juego en la relación formativa". En: Revista Argentina de Educación Superior. Red Argentina de Posgrados en Educación Superior. № 6. Mayo, 2013. Edición digital: http://www.raes.org.ar/revistas/raes6_conf5.pdf

Mancovsky, V. (2014a) - «Traces d'une relation formative » En Revue CLIOPSY (Clinique d'orientation psychanalytique dans le champ de l'éducation et de la formation) $\mathrm{N}^{\circ}$ 12. octobre, 2014. Version digital: http://www.revuecliopsy.fr/n-12-octobre-2014/

Mancovsky, V. (2014b) "Un director de tesis se va haciendo, no se forma de antemano... La dirección no se aprende de la noche a la mañana... Reflexiones en torno a la relación formativa entre director de tesis y tesista en el nivel del doctorado". Ponencia presentada en el IV Congreso Internacional "Nuevas Tendencias en la Formación Permanente del Profesorado". Universidad Nacional de Tres de Febrero, la Universidad Pedagógica, la Universidad de Barcelona (España).

Mancovsky, V. y moreno Bayardo, M. G. (2015) La formación para la investigación en el posgrado. Buenos Aires. Ed. Novedades Educativas.

\section{Notas:}

${ }^{\text {i } E s ~ n e c e s a r i o ~ a c l a r a r ~ q u e ~ t o d o ~ n i v e l ~ e d u c a t i v o ~ p u e d e ~ s e r ~ l e i ́ d o ~ d e s d e ~ e s t e ~ e n t r a m a d o ~ d e ~ d i m e n s i o n e s ~ d e ~}$ análisis. Lo particularmente relevante del doctorado es su modo de haber sido creado e institucionalizado a partir de la Ley de Educación Superior de 1995. Varios autores describen y señalan su funcionamiento como un "microcosmos", por fuera mismo de la lógica institucional y normativa del nivel universitario. Los valiosos aportes de M. de la Fare y S. Lenz (2012) describen detalladamente esta "marca de nacimiento" dentro de la problemática del posgrado en la Argentina desde la creación de dicha ley.

ii Las investigaciones que dirigí sobre esta temática comprendieron dos etapas: en el período 2010-2011, el proyecto se tituló Hacia una Pedagogía de la Formación doctoral: un estudio de caso. En el 2012-2013, la investigación llevó por título: Pedagogía de la Formación doctoral: estudio empírico y avances teóricos. Ambos fueron encuadrados en la programación de la Secretaría de Investigación y Desarrollo de la Universidad Nacional de Tres de Febrero (UNTREF). En el primer trabajo, el grupo de investigación estuvo constituido por Bettina Checchia, Mirta Fabris y Lucas Krotsch. Los dos últimos integrantes dejaron de participar en el segundo período de trabajo.

Entre el período 2014-2015, realicé y dirigí otro proyecto con un nuevo equipo de investigadores interesados en el estudio de las tutorías de grado y de posgrado. Llevó por título: Las representaciones sociales del tutor universitario: un enfoque interdisciplinario desde la sociología, la psicología y la pedagogía. El mismo fue concretado en el marco del Programa de Investigaciones de la Secretaría de Ciencia y Técnica de la Universidad Nacional de Lanús. El equipo estuvo conformado por Esteban Pintos Andadre y María Isabel Boniscontro. Contó con la colaboración de dos profesores de la Facultad de Derecho de la Universidad de la República (Uruguay). Parte del trabajo empírico fue realizado con directores de tesis de esta institución.

Actualmente, continúo en la dirección de un nuevo proyecto con un grupo de colegas investigadoras de la UNTREF: Cristina Tommasi, Trinidad Cocha y Gabriela Furlan. El proyecto se titula: "Estudio de las interacciones formativas de los tesistas en el marco de una Pedagogía Doctoral" y se encuadra en la Programación 2016-2017 de la Secretaría de Investigación y Desarrollo de dicha universidad.

iii Como contrapunto, J. Beillerot, un reconocido especialista francés que enriqueció el campo de la Pedagogía de la Formación con importantes aportes e investigaciones, plantea una sugerente reflexión para analizar la cualidad de lo político en el debate de la pedagogía en la universidad. Este autor afirma: 
“... el problema de enseñar o formar tiene una dimensión política muy fuerte. Tengo la costumbre de decir que la pedagogía, es decir ese arte que consiste en ayudar al sujeto a aprender, es necesario y útil si se piensa en términos de democracia". (Beillerot, 1996: 21). Considero que, en los tiempos que corren, sería interesante avanzar con este posicionamiento a favor de la pedagogía desde el planteo democrático de la formación universitaria de grado y de posgrado.

iv Las imágenes presentadas forman parte de la serie de esculturas de Leo Vinci, escultor argentino, que se llevó a cabo en la Universidad Nacional de Lanús, bajo el nombre de "Cien creadores para miles de amaneceres" en el Espacio de Arte de esa institución en abril, 2010. 